\title{
Analytic solutions for Long's equation and its generalization
}

\author{
Mayer Humi \\ Correspondence to: Mayer Humi (mhumi@wpi.edu) \\ Received: 27 June 2017 - Discussion started: 10 July 2017 \\ Revised: 9 October 2017 - Accepted: 27 October 2017 - Published: 5 December 2017
}

Department of Mathematical Sciences, Worcester Polytechnic Institute, 100 Institute Road, Worcester, MA 01609, USA

\begin{abstract}
Two-dimensional, steady-state, stratified, isothermal atmospheric flow over topography is governed by Long's equation. Numerical solutions of this equation were derived and used by several authors. In particular, these solutions were applied extensively to analyze the experimental observations of gravity waves. In the first part of this paper we derive an extension of this equation to non-isothermal flows. Then we devise a transformation that simplifies this equation. We show that this simplified equation admits solitonic-type solutions in addition to regular gravity waves. These new analytical solutions provide new insights into the propagation and amplitude of gravity waves over topography.
\end{abstract}

\section{Introduction}

Two-dimensional steady-state flow of an isothermal, incompressible stratified fluid over topography is modeled by Long's equation (Long, 1953, 1954, 1955, 1959). A generalization of this equation to three-dimensional flows has appeared in the literature (Akylas and Davis, 2001). However, in the following we restrict our discussion to two dimensions.

Numerical solutions of Long's equation for base flow without shear over simple terrain, which consists of one hill, were derived and analyzed in the literature by several authors (Drazin, 1961; Yih, 1967; Drazin and Moore, 1967; Lily and Klemp, 1979; Smith, 1980, 1989; Peltier and Clark, 1983; Durran, 1992; Smith and Kruse, 2017).

In these studies it was usual to approximate the BruntVäisälä frequency by a constant or a step function. In addition, two physical parameters which control the stratification and dispersive effects of the atmosphere were set to zero. Under these approximations, one of the leading second-order derivatives in Long's equation drops out. Moreover, the equation becomes linear (the nonlinear terms disappear). In this singular limit Long's equation reduces to that of a linear harmonic oscillator over the computational domain. The impact of these approximations on the validity of the solution was analyzed in depth in the literature (Smith, 1980, 1989; Peltier and Clark, 1983). These studies demonstrated that these approximations set limits on the physical applicability of these solutions.

Solutions of Long's equation were also used as a framework for the examination and study of experimental data on gravity waves (Shutts et al., 1988, 1994; Fritts and Alexander, 2003; Jumper et al., 2004; Vernin et al., 2007; Richter et al., 2010; Geller et al., 2013). In all of these studies it was assumed that the base flow is shearless. However, this assumption is incorrect, in general, and is not justified by the experimental data. (For a comprehensive list of references, see Yih, 1980, Baines, 1995, and Nappo, 2012.)

A new method to derive analytic solutions of Long's equation was initiated by the present author in Humi (2004, 2007, $2009,2010,2015)$. It was demonstrated that Long's equation can be simplified for shearless base flow with mild assumptions about the nonlinear terms. In this framework we were able to identify the "slow variable" in Long's equation. This variable controls the emergence of nonlinear oscillations in this equation. In addition we proved the existence of selfsimilar solutions and derived a formula for the attenuation of the gravity wave amplitude with height. These results follow from the general properties of Long's equation and the nonlinear terms present in this equation.

We considered the effect that shear in the base flow has on the generation of gravity waves and their amplitude in Humi (2006). A new form of Long's equation in which the stream function is replaced by the atmospheric density was derived in Humi (2007). Finally a generalization of Long's equation to time-dependent flows appeared in Humi (2015). 
It obvious however that atmospheric flows over topography are not isothermal in general (see Miglietta and Rotunno, 2014; Richter et al., 2010; Smith and Kruse, 2017, and their bibliography). With this motivation we derive, in the first part of this paper, an extension of this equation to include non-isothermal flows with free convection. This extension of Long's equation is new.

The novel part of the current paper consists of a sequence of transformations which linearize Long's equation and lead to an analytic form of the solution without scarifying any of the physical contents of this equation. In particular, we demonstrate that there exist "solitonic-type solutions" to this equation in addition to regular gravity waves. These types of solutions have never appeared in the literature before. The solutions presented also show how the amplitudes of the gravity waves depend on the height. The presentations in Sect. 2.1 and 2.3 are made in order to put the new novel aspects of this paper in context and to give the reader a sense of their importance. The bulk of the paper, which comprises Sects. 2.2, 3 and 4 , presents completely new results which have never appeared in the literature before.

The plan of the paper is as follows: in Sects. 2.1 and 2.3 we present an overview of the derivation of the isothermal Long equation and the approximations that are made for its numerical solutions. In Sect. 2.2 we derive the corresponding Long equation for flows with free convection. In Sect. 3 we introduce a transformation which (essentially) linearizes the equation for the perturbation from the base flow. Section 4 discusses the application of this transformation to a flow with shear and presents an analytic solution for this flow. We end with some conclusions in Sect. 5.

\section{Derivation of Long's equation}

In the first part of this section we provide a short overview of the (classical) isothermal Long equation and in the second part we generalize this equation to include free convection.

\subsection{Isothermal Long equation}

In two dimensions $(x, z)$ the flow of a steady isothermal, inviscid and incompressible stratified fluid is modeled by the following equations:

$u_{x}+w_{z}=0$,

$u \rho_{x}+w \rho_{z}=0$,

$\rho\left(u u_{x}+w u_{z}\right)=-p_{x}$,

$\rho\left(u w_{x}+w w_{z}\right)=-p_{z}-\rho g$.

In these equations, subscripts denote differentiation with respect to the subscripted variable, $\boldsymbol{u}=(u, w)$ is the fluid velocity, $p$ denotes the pressure, $\rho$ denotes the density and $g$ is the acceleration of gravity.
To non-dimensionalize Eqs. (1)-(4), we introduce the following scaled variables:

$\bar{x}=\frac{x}{L}, \bar{z}=\frac{N_{0}}{U_{0}} z, \quad \bar{u}=\frac{u}{U_{0}}, \quad \bar{w}=\frac{L N_{0}}{U_{0}^{2}} w$,

$\bar{\rho}=\frac{\rho}{\overline{\rho_{0}}}, \quad \bar{p}=\frac{N_{0}}{g U_{0} \overline{\rho_{0}}} p$.

In these equations $L$ represents a characteristic length, $U_{0}$ is the free stream velocity, and $\overline{\rho_{0}}$ is the averaged base density which is considered to be a constant. $N_{0}^{2}$ represents an averaged value of the Brunt-Väisälä frequency which is defined as

$N^{2}=-\frac{g}{\rho_{0}} \frac{\mathrm{d} \rho_{0}}{\mathrm{~d} z}$

where $\rho_{0}(z)$ is the base density.

Using these new variables, Eqs. (1)-(4) take the following form (the bars were dropped for brevity):

$u_{x}+w_{z}=0$,

$u \rho_{x}+w \rho_{z}=0$

$\beta \rho\left(u u_{x}+w u_{z}\right)=-p_{x}$,

$\beta \rho\left(u w_{x}+w w_{z}\right)=-\mu^{-2}\left(p_{z}+\rho\right)$,

where

$\mu=\frac{U_{0}}{N_{0} L}$,

$\beta=\frac{N_{0} U_{0}}{g}$.

In these equations $\mu$ is the longwave parameter which controls dispersive effects or equivalently the deviation from the hydrostatic approximation. When $\mu=0$ the hydrostatic approximation is fully satisfied (Smith, 1980, 1989). The coefficient $\beta$ is the Boussinesq parameter (Baines, 1995; Nappo, 2012), which controls stratification effects (assuming $U_{0} \neq$ $0)$.

Equation (7) implies that it is possible to introduce a stream function $\psi$ so that

$u=\psi_{z}, w=-\psi_{x}$.

Using this definition of $\psi$, it is possible to rewrite Eq. (8) as

$J\{\rho, \psi\}=0$.

The symbol $J(f, g)$ is defined for any two smooth functions $f$ and $g$ as

$J\{f, g\}=\frac{\partial f}{\partial x} \frac{\partial g}{\partial z}-\frac{\partial f}{\partial z} \frac{\partial g}{\partial x}$.

It is easy to show that when $J(f, g)=0$ it is possible to express each of these functions in terms of the other (Yih, 
1980). It follows then from Eq. (14) that the functions $\rho$ and $\psi$ are dependent on each other. This means that one can express $\rho$ as $\rho(\psi)$ or $\psi$ as $\psi(\rho)$.

Using Eq. (13) one can rewrite the momentum Eqs. (9) and (10) in terms of $\psi$.

$\beta \rho\left(\psi_{z} \psi_{z x}-\psi_{x} \psi_{z z}\right)=-p_{x}$

$\beta \rho\left(-\psi_{z} \psi_{x x}+\psi_{x} \psi_{x z}\right)=-\mu^{-2}\left(p_{z}+\rho\right)$

To eliminate $p$ from Eqs. (16) and (17), we multiply Eq. (17) by $\mu^{2}$ and then differentiate Eqs. (16) and (17) with respect to $z$ and $x$, respectively, and subtract. We obtain

$$
\begin{aligned}
& \rho_{z}\left(\psi_{z} \psi_{z x}-\psi_{x} \psi_{z z}\right)+\rho\left(\psi_{z} \psi_{z x}-\psi_{x} \psi_{z z}\right)_{z} \\
& \quad-\beta \mu^{2} \rho_{x}\left(-\psi_{z} \psi_{x x}+\psi_{x} \psi_{x z}\right) \\
& \quad-\beta \mu^{2} \rho\left(-\psi_{z} \psi_{x x}+\psi_{x} \psi_{x z}\right)_{x}=\rho_{x} .
\end{aligned}
$$

Using Eq. (14) and the fact that

$\rho_{x}=\rho_{\psi} \psi_{x}, \rho_{z}=\rho_{\psi} \psi_{z}$,

we can eliminate $\rho$ from Eq. (18) and obtain after some algebra

$$
\begin{aligned}
& J\left\{\psi_{z z}+\mu^{2} \psi_{x x}, \psi\right\} \\
& -N^{2}(\psi) J\left\{\frac{\beta}{2}\left(\psi_{z}^{2}+\mu^{2} \psi_{x}^{2}\right), \psi\right\}=N^{2} J\{z, \psi\}
\end{aligned}
$$

where

$N^{2}(\psi)=-\frac{\rho_{\psi}}{\beta \rho}$

is the non-dimensional Brunt-Väisälä frequency which is (by definition) a function of $\psi$.

As a result we obtain the following equation for $\psi$ (Baines, 1995; Nappo, 2012):

$\psi_{z z}+\mu^{2} \psi_{x x}-N^{2}(\psi)\left[z+\frac{\beta}{2}\left(\psi_{z}^{2}+\mu^{2} \psi_{x}^{2}\right)\right]=G(\psi)$.

Equation (22) is referred to in the literature as "Long's equation", but it was derived first by Dubreil-Jacotin (DubreilJacotin, 1935).

In Eq. (22), $G(\psi)$ is a function that has to be determined from the base flow. To do so we consider Eq. (22) at $x=-\infty$ and assume that the base flow is a function of $z$ only. Then we express the left-hand side of Eq. (22) in terms of $\psi$ only to determine $G(\psi)$. (Here we assumed, following Yih, 1967, 1980, and Baines, 1995, that the disturbances from the base flow do not propagate upstream.)

For example, if we consider a shearless base flow with $u(-\infty, z)=1$, then

$\psi(-\infty, z)=z$

and

$G(\psi)=-N^{2}(\psi)\left(\frac{\beta}{2}+\psi\right)$.
Equation (22) becomes

$$
\begin{aligned}
\psi_{z z} & +\mu^{2} \psi_{x x} \\
- & N^{2}(\psi)\left[z-\psi+\frac{\beta}{2}\left(\psi_{z}^{2}+\mu^{2} \psi_{x}^{2}-1\right)\right]=0 .
\end{aligned}
$$

It follows from this example that different base flows at $x=-\infty$ will yield different functional forms of $G(\psi)$.

We consider now a perturbation $\eta$ from a shearless base flow $u(-\infty, z)=1$, viz.

$\eta=\psi-z$

Substituting this expression into Eq. (22) leads to

$\eta_{z z}+\mu^{2} \eta_{x x}-\frac{N^{2} \beta}{2}\left(\eta_{z}^{2}+\mu^{2} \eta_{x}^{2}+2 \eta_{z}\right)+N^{2} \eta=0$.

\subsection{Long's equation with free convection}

When the flow is not isothermal, Eq. (4) has to be modified as follows:

$\rho\left(u w_{x}+w w_{z}\right)=-p_{z}-\gamma T \rho g$,

where $T$ is the temperature and $\gamma$ is the thermal expansion coefficient of the fluid. Moreover, an equation for the temperature has to be added:

$\boldsymbol{u} \cdot \nabla T=\chi \nabla^{2} T$,

where $\chi$ is its thermometric conductivity. These equations hold under the assumption that

$\frac{g h}{c^{2}} \ll \gamma T_{0}$

where $h$ is the fluid column height, $c$ is the velocity of sound in the fluid and $T_{0}$ is the characteristic temperature difference.

We can non-dimensionalize these equations using Eq. (5) with the addition of

$\bar{T}=\frac{T}{T_{0}}$

(as in the previous subsection we drop the bars). Equations (28) and (29) become

$\beta \rho\left(u w_{x}+w w_{z}\right)=-\mu^{-2}\left(p_{z}+\gamma T \rho\right)$,

$\boldsymbol{u} \cdot \nabla T=\frac{1}{P e} \nabla^{2} T$,

where $P e=\frac{U_{0} L}{\chi}$ is the Peclet number. 
Using Eq. (7) to introduce a stream function $\psi$, the momentum Eqs. (9) and (30) become

$\beta \rho\left(\psi_{z} \psi_{z x}-\psi_{x} \psi_{z z}\right)=-p_{x}$,

$\beta \rho\left(-\psi_{z} \psi_{x x}+\psi_{x} \psi_{x z}\right)=-\mu^{-2}\left(p_{z}+\gamma T \rho\right)$.

Using the same strategy as in the previous subsection to eliminate $p$ from these equations leads to

$$
\begin{aligned}
& \rho_{z}\left(\psi_{z} \psi_{z x}-\psi_{x} \psi_{z z}\right)+\rho\left(\psi_{z} \psi_{z x}-\psi_{x} \psi_{z z}\right)_{z} \\
& \quad-\mu^{2} \rho_{x}\left(-\psi_{z} \psi_{x x}+\psi_{x} \psi_{x z}\right) \\
& \quad-\mu^{2} \rho\left(-\psi_{z} \psi_{x x}+\psi_{x} \psi_{x z}\right)_{x}=\frac{\gamma}{\beta}(T \rho)_{x} .
\end{aligned}
$$

If the diffusion processes in Eq. (31) can be ignored, i.e., $\left|\frac{1}{P e} \nabla^{2} T\right| \ll 1$, then this equation can approximated by

$J\{T, \psi\}=0$;

i.e., $T=T(\psi)$. Furthermore, since $\rho=\rho(\psi)$, it follows that

$(T \rho)_{x}=-J\{z, T \rho\}=-\frac{\partial(T \rho)}{\partial \psi} J\{z, \psi\}$.

Using Eqs. (14), (35) and (19), we can eliminate $\rho$ from Eq. (34) and obtain, after some algebra,

$$
\begin{aligned}
& J\left\{\psi_{z z}+\mu^{2} \psi_{x x}, \psi\right\} \\
& -N^{2}(\psi) J\left\{\frac{\beta}{2}\left(\psi_{z}^{2}+\mu^{2} \psi_{x}^{2}\right), \psi\right\}=M^{2} J\{z, \psi\}
\end{aligned}
$$

where

$$
M^{2}=-\frac{\gamma}{\beta \rho}(T \rho)_{\psi} .
$$

Using these definitions, it follows that

$$
\begin{aligned}
\psi_{z z}+\mu^{2} \psi_{x x}-N^{2}(\psi) \frac{\beta}{2}\left(\psi_{z}^{2}+\mu^{2} \psi_{x}^{2}\right) \\
-M^{2}(\psi) z=G(\psi) .
\end{aligned}
$$

Eq. (39) can be considered a generalized form of Long's equation which includes the effects of free convection. It contains two parameters $N^{2}$ and $M^{2}$. The additional parameter $M^{2}$ controls the change in the temperature profile in the flow.

The function $G(\psi)$ in Eq. (39) can be determined using the same strategy as before. Thus, if $\psi(-\infty, z)$ is given by Eq. (23), then

$$
G(\psi)=-N^{2}(\psi) \frac{\beta}{2}-M^{2}(\psi) \psi
$$

and Eq. (39) becomes

$$
\begin{gathered}
\psi_{z z}+\mu^{2} \psi_{x x}-N^{2}(\psi) \frac{\beta}{2}\left(\psi_{z}^{2}+\mu^{2} \psi_{x}^{2}-1\right) \\
-M(\psi)^{2}(z-\psi)=0 .
\end{gathered}
$$

For a perturbation $\eta=\psi-z$, from a base flow $u(-\infty, z)=1$, we obtain from Eq. (39)

$\eta_{z z}+\mu^{2} \eta_{x x}-\frac{N^{2} \beta}{2}\left(\eta_{z}^{2}+\mu^{2} \eta_{x}^{2}+2 \eta_{z}\right)+M^{2} \eta=0$.

\subsection{Boundary conditions and approximations}

We consider here numerical solutions of Long's equation over an unbounded domain with a general base flow. The topography of the domain is represented by a function $h(x)$ whose maximum height is $H$. The boundary conditions that are imposed on the stream function $\psi$ are

$\psi(-\infty, z)=\psi_{0}(z)$,

$\psi(x, \tau h(x))=$ constant, $\tau=\frac{H N_{0}}{U_{0}}$.

The constant in Eq. (44) which represents the value of the stream line over the topography $h(x)$ is (usually) set to zero.

To determine the proper boundary condition on $\psi(\infty, z)$, we note that Long's equation has no dissipation terms. Therefore radiation boundary conditions have to be imposed on $\psi$ in this limit. Similarly it is appropriate to impose radiation boundary conditions on $\psi(x, \infty)$ (Durran, 1992).

When $|\tau| \ll 1$ the boundary condition (44) can be approximated (using Eq. 26) by

$\eta(x, 0)=-\tau h(x)$.

When $N$ and $M$ are set to a constant, Eqs. (27) and (42) become invariant with respect to translations in $x, z$. This implies that these equations admit self-similar solutions in the form $\eta=f(m x+n z)$ (Humi, 2004). These solutions represent gravity waves that are generated by the flow over the topography.

To compute numerical solutions for the perturbation $\eta$ over topography, it has been common in the literature to consider Eq. (27) in the limits $\mu=0$ and $\beta=0$ (Durran, 1992; Lily and Klemp, 1979). In addition, $N$ is set to a constant or a step function over the computational domain.

In these limits Eq. (27) becomes a linear equation:

$\eta_{z z}+N^{2} \eta=0$.

The limit $\beta=0$ can be obtained by letting either $N_{0} \rightarrow 0$ or $U_{0} \rightarrow 0$. For the stratification to persist, one has to assume that the limit $\beta=0$ is obtained as $U_{0} \rightarrow 0$.

Equation (46) is a singular limit of Eq. (27). This is due to the fact that one of the leading second-order derivatives drops when $\mu=0$. Moreover, the nonlinear terms in this equation drop out when $\beta=0$. The approximate solutions that are derived from Eq. (46) and their physical limitations have been considered extensively in the literature (Drazin and Moore, 1967; Durran, 1992; Humi, 2004, 2006). It was found that strong restrictions have to be imposed on the validity of these solutions even under the assumption that the base flow is shearless. However, these approximations and the solutions that are derived from Eq. (46) are used routinely in the analysis of experimental atmospheric data (Shutts et al., 1988; Baines, 1995; Jumper et al., 2004; Vernin et al., 2007).

The general solution of Eq. (46) is of the form

$\eta(x, z)=q(x) \cos (N z)+p(x) \sin (N z)$. 
The functions $p(x)$ and $q(x)$ have to satisfy the boundary conditions derived from Eq. (44) and the radiation boundary conditions. To satisfy the radiation boundary conditions, $p(x)$ and $q(x)$ have to satisfy (Baines, 1995; Nappo, 2012) $p(x)=H[q(x)]$, where $H[q(x)]$ is the Hilbert transform of $q(x)$.

To satisfy the boundary condition on the terrain, one has to solve the following integral equation (Drazin, 1961; Lily and Klemp, 1979; Durran, 1992):

$$
q(x) \cos (\tau N f(x))+H[q(x)] \sin (\tau N f(x))=-\tau h(x) .
$$

\section{Reductions and transformations}

To begin with we observe that in Eqs. (22), (39), (27), and (42) one can suppress the appearance of the parameter $\mu^{2}$ $(\mu \neq 0)$ by applying the transformation $x=\mu \bar{x}$. Performing this transformation and assuming that $N$ and $M$ are constants, these equations become invariant with respect to translations in $x$ and $z$. As a result they have solutions of the form $\eta=f(k \bar{x}+m z)$ (Humi, 2004). These are gravity waves that are generated by the atmospheric flow over the terrain.

Equation (27) becomes

$\eta_{z z}+\eta_{x x}-\alpha^{2}\left(\eta_{z}^{2}+\eta_{x}^{2}+2 \eta_{z}\right)+N^{2} \eta=0$

where

$\alpha^{2}=\frac{N^{2} \beta}{2}$.

Similarly, Eq. (42) becomes

$\eta_{z z}+\eta_{x x}-\alpha^{2}\left(\eta_{z}^{2}+\eta_{x}^{2}+2 \eta_{z}\right)+M^{2} \eta=0$.

To these equations we apply the transformation

$\phi=e^{-\alpha^{2} \eta}-1$.

Remark: the mathematical "inspiration" for this transformation comes from somewhat similar transformations which linearize the Ricatti and Burger equations. From a physical point of view the motivation comes from the desire to replace the nonlinearities due to the derivatives of $\eta$ in Eq. (50) with expressions that correspond to $\eta$ itself. This replacement will enable us to make approximations which are based on physical insights.

Equations (49) and (50), respectively, become

$\nabla^{2} \phi-2 \alpha^{2} \frac{\partial \phi}{\partial z}+N^{2}(1+\phi) \ln (1+\phi)=0$,

$\nabla^{2} \phi-2 \alpha^{2} \frac{\partial \phi}{\partial z}+M^{2}(1+\phi) \ln (1+\phi)=0$.

Since $\left|\alpha^{2} \eta\right| \ll 1$ it follows that $|\phi| \ll 1$, and we can make the approximation $\ln (1+\phi) \approx \phi$. Equations (52) and (53) be- come

$\nabla^{2} \phi-2 \alpha^{2} \frac{\partial \phi}{\partial z}+N^{2}(1+\phi) \phi=0$,

$\nabla^{2} \phi-2 \alpha^{2} \frac{\partial \phi}{\partial z}+M^{2}(1+\phi) \phi=0$.

To simplify Eqs. (54) and (55), we introduce the transformation

$\phi=e^{\alpha^{2} z} y$.

Equation (54) becomes

$\nabla^{2} y+\left(N^{2}-\alpha^{4}\right) y+N^{2} e^{\alpha^{2} z} y^{2}=0$.

If $\left|\alpha^{2} z\right| \ll 1$ (in the domain of interest), we can approximate this equation by

$\nabla^{2} y+\left(N^{2}-\alpha^{4}\right) y+N^{2} y^{2}=0$.

This equation has an analytic closed form solution

$y=\frac{3\left(N^{2}-\alpha^{4}\right)}{n^{2}}\left[\tanh ^{2}\left(C_{1}+C_{2} x-i v z\right)-1\right]$

where

$v^{2}=N^{2}-\alpha^{4}+4 C_{2}^{2}$

and $C_{1}$ and $C_{2}$ are integration constants.

Equation (59) represents solutions to a nonlinear equation for $y$ (and hence $\eta$ ). Since there is no superposition principle for these solutions, Eq. (59) represents a new "soliton-type solution" for $\eta$ (in Eq. 49). Using the approximation $e^{\alpha^{2} z}=$ $1+\alpha^{2} z$, this solution for $\phi$ (using Eq. 56) satisfies Eq. (52) up to terms on order $\alpha^{2}$.

If $\alpha^{2} z$ is not small, one can approximate $e^{\alpha^{2} z}$ by $1+\alpha^{2} z$ and use a perturbation expansion $y=y_{0}+\alpha^{2} y_{1}$ to compute $y_{1}$ (numerically).

Similar treatment can be applied to Eq. (55).

\subsection{Linearized equations and solutions}

To obtain a real solution for $\phi$, we neglect the $\phi^{2}$ term in Eqs. (54) and (55) as being of second order. These approximations linearize Eqs. (52) and (53) and yield (respectively)

$$
\begin{aligned}
& \nabla^{2} \phi-2 \alpha^{2} \frac{\partial \phi}{\partial z}+N^{2} \phi=0, \\
& \nabla^{2} \phi-2 \alpha^{2} \frac{\partial \phi}{\partial z}+M^{2} \phi=0 .
\end{aligned}
$$

These equations can be solved using separation of variables. Due to the similarity between Eqs. (60) and (61) we discuss henceforth the solution procedure for Eq. (60) only. 
If we substitute $\phi=f(x) g(z)$ into Eq. (60) and perform separation of variables, we obtain the following equations for $f$ and $g$ :

$\frac{\mathrm{d}^{2} f}{\mathrm{~d} x^{2}}+\omega^{2} f=0$,

$\frac{\mathrm{d}^{2} g}{\mathrm{~d} x^{2}}-2 \alpha^{2} \frac{\mathrm{d} g}{\mathrm{~d} z}+\left(N^{2}-\omega^{2}\right) g=0$.

Hence,

$$
\begin{aligned}
& f_{\omega}=A(\omega) e^{i \omega x}+B(\omega) e^{-i \omega x}, \\
& g_{\omega}=e^{\alpha^{2} z}\left(C_{1}(\omega) e^{i \nu z}+C_{2}(\omega) e^{-i \nu z}\right),
\end{aligned}
$$

where $C_{1}$ and $C_{2}$ are constants and $v=\sqrt{N^{2}-\alpha^{4}-\omega^{2}}$. Hence for a wave to exist (in the $z$-direction) we must have $N^{2} \geq \alpha^{4}+\omega^{2}$. In addition the wave amplitude increases with height by a factor of $e^{\alpha^{2} z}$.

Similarly to Eq. (61) we obtain the same expression for $f(x)$ and

$g_{\omega}=e^{\alpha^{2} z}\left(C_{3}(\omega) e^{i \lambda z}+C_{4}(\omega) e^{-i \lambda z}\right)$

where $\lambda=\sqrt{M^{2}-\alpha^{4}-\omega^{2}}$.

The general solution of Eq. (60) can be written as

$$
\begin{aligned}
\phi & = \\
& e^{\alpha^{2} z} \int\left[\left(D_{1}(\omega) e^{i(v z+\omega x)}+D_{2}(\omega) e^{-i(\nu z+\omega x)}\right] \mathrm{d} \omega\right. \\
& +e^{\alpha^{2} z} \int\left[D_{3}(\omega) e^{i(v z-\omega x)}+D_{4}(\omega) e^{-i(v z-\omega x)}\right] \mathrm{d} . \omega
\end{aligned}
$$

Since the exponents multiplying $D_{1}$ and $D_{2}$ are conjugates, it follows that for $\phi$ to be real we must have $\overline{D_{1}}=D_{2}$ (where the bar stands for complex conjugation). Similarly we must have $\overline{D_{3}}=D_{4}$.

The radiation boundary condition at $z \rightarrow \infty$ requires that the group velocity of the outgoing wave is positive. For a hydrostatic flow the dispersion relation is given by

$\lambda(\omega)=\omega-\frac{\operatorname{sgn}(v) N \omega}{v}$

and the group velocity is

$v_{g}=\frac{\partial \lambda}{\partial v}=\frac{\operatorname{sgn}(v) N \omega}{v^{2}}$.

Hence $v_{g}>0$ if $\nu \omega>0$.

Since the integration in Eq. (67) is over positive $\omega$, it follows then that the last two terms in this equation must be zero $(v \omega<0)$.

To satisfy boundary condition (45), we observe (using Eq. 51) that

$\eta=-\frac{\ln (1+\phi)}{\alpha^{2}}$.
Hence the boundary condition (45) becomes

$\phi(x, 0)=e^{\alpha^{2} \tau h(x)}-1 \approx \alpha^{2} \tau h(x)$.

It follows then from Eq. (67) that

$$
\begin{aligned}
& \int 2 \operatorname{Re} D_{1}(\omega) \cos (\omega x) \mathrm{d} \omega \\
& -\int 2 \operatorname{Im} D_{1}(\omega) \sin (\omega x) \mathrm{d} \omega=\alpha^{2} \tau h(x) .
\end{aligned}
$$

This can be satisfied by standard Fourier integral expansion of $h(x)$.

The special case $\mu=0$ was treated in detail in Humi (2004).

\subsection{Application}

To examine the application of the formulas derived above, we consider the flow over a "witch of Agnesi" hill where the height of the topography is given by

$h(x)=\frac{a^{2}}{\left(a^{2}+x^{2}\right)}$.

The Fourier integral expansion of $h(x)$ is

$h(x)=\int_{0}^{\infty} A(\omega) \cos (\omega x) \mathrm{d} \omega$

where

$A(\omega)=a e^{-a \omega}$.

Using Eq. (70), this implies that $\operatorname{Im} D_{1}=0$ and

$D_{1}(\omega)=\frac{\alpha^{2} \tau A(\omega)}{2}$.

Substituting this result in Eq. (67) yields

$\phi=e^{\alpha^{2} z}\left\{\int\left[D_{1}(\omega) e^{i(\nu z+\omega x)}+D_{2}(\omega) e^{-i(\nu z+\omega x)}\right] \mathrm{d} \omega\right\}$.

Hence,

$\phi=\alpha^{2} \tau e^{\alpha^{2} z} \int e^{-a \omega} \cos (\nu z+\omega x) \mathrm{d} \omega$.

From this expression we can compute $\eta$ using Eq. (68). Figure 1 displays the solution for $\eta$ for isothermal flow with $N=1.5, \beta=0.01, a=1$, and $\tau=1$. Figure 2 displays the solution for $\eta$ for non-isothermal flow with the same parameters as in Fig. 1 but with $M=2$. These plots demonstrate the dependence of the gravity wave amplitude on the height and the impact that non-isothermal flow might have on the direction and amplitude of the wave. 


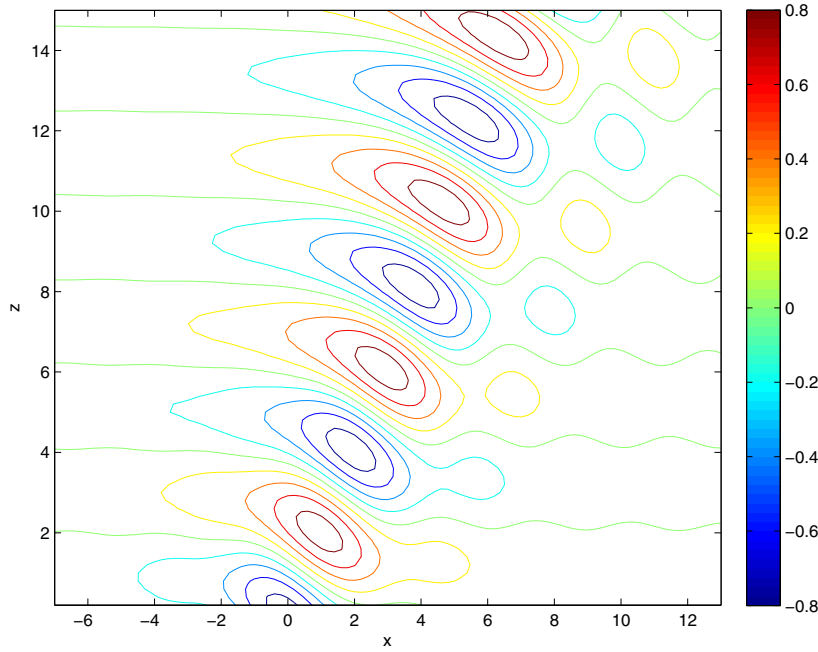

Figure 1. Contour plot of $\eta$ for isothermal flow over a topography.

\section{Solutions with shear}

We consider here a base flow with $u=z$, i.e., $\psi(-\infty, z)=$ $z^{2}$. Using Eq. (22) to compute $G(\psi)$, we find that

$G(\psi)=2-N^{2}\left(\psi^{1 / 2}+2 \beta \psi\right)$.

Long's equation (22) (with $\mu \neq 0$ ) becomes

$$
\begin{aligned}
\psi_{z z} & +\mu^{2} \psi_{x x}-N^{2}(\psi)\left[z+\frac{\beta}{2}\left(\psi_{z}^{2}+\mu^{2} \psi_{x}^{2}\right)\right] \\
& =2-N^{2}\left(\psi^{1 / 2}+2 \beta \psi\right) .
\end{aligned}
$$

Applying the transformation $\bar{x}=\frac{x}{\mu}$, we obtain (after dropping the bars)

$$
\begin{aligned}
& \left(\psi_{z z}-\alpha^{2} \psi_{z}^{2}\right)+\left(\psi_{x x}-\alpha^{2} \psi_{x}^{2}\right)-N^{2} z \\
& \quad=2-N^{2}\left(\psi^{1 / 2}+2 \beta \psi\right) .
\end{aligned}
$$

For a perturbation $\eta$ from the base flow, i.e., $\psi=z^{2}+\eta$, we obtain the following equation (where the square root was linearized assuming $|\eta| \ll 1)$ ):

$$
\begin{aligned}
\eta_{z z} & -4 \alpha^{2} z \eta_{z}-\alpha^{2}\left(\eta_{z}\right)^{2}+\eta_{x x} \\
& -\alpha^{2}\left(\eta_{x}\right)^{2}+\left(4 \alpha^{2}+\frac{N^{2}}{2 z}\right) \eta=0 .
\end{aligned}
$$

We now introduce the transformation

$\phi=e^{-\alpha^{2} \eta}-b$

where $b \neq 0$ is a parameter to be determined later. Applying this transformation to Eq. (79) and making the approximation $\ln (b+\phi)=\ln (b)+\frac{\phi}{b}$ (assuming $\left.|\phi| \ll b\right)$ leads to the

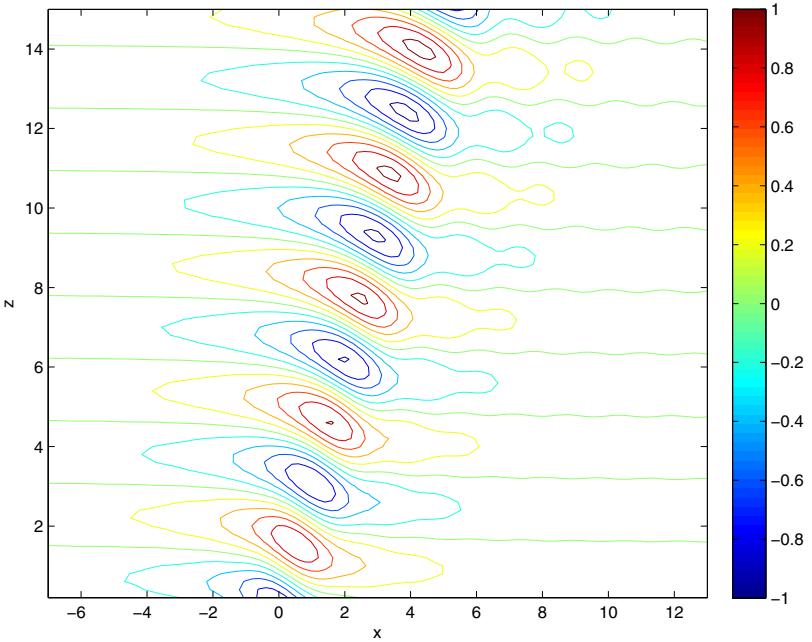

Figure 2. Contour plot of $\eta$ for non-isothermal flow over a topography.

following:

$$
\begin{aligned}
& 2 b z \phi_{z z}+2 b z \phi_{x x}-8 b \alpha^{2} z^{2} \phi_{z} \\
& \quad+\left(8 \alpha^{2} z+N^{2}\right)\left[\phi^{2}+b(\ln (b)+1) \phi+b^{2} \ln (b)\right]=0 .
\end{aligned}
$$

Dropping the nonlinear term in $\phi^{2}$ and letting $b=e^{-1}$ (to suppress the term containing $\phi$ ), Eq. (81) becomes

$2 z \phi_{z z}+2 z \phi_{x x}-8 \alpha^{2} z^{2} \phi_{z}-e^{-1}\left(8 \alpha^{2} z+N^{2}\right)=0$.

A particular solution $\phi_{p}$ of this (linear) equation is (Abramowitz and Stegun, 1974)

$$
\begin{aligned}
\phi_{p} & =-\frac{1}{4} \int e^{2 \alpha^{2} z^{2}-1}[-4 \alpha \sqrt{2 \pi} \operatorname{erf}(\sqrt{2} \alpha z) \\
& \left.+N^{2} \Gamma\left(0,2 \alpha^{2} z^{2}\right)\right] \mathrm{d} z .
\end{aligned}
$$

The homogeneous part of Eq. (82) can be solved by separation of variables, viz. $\phi=f(x) g(z)$, where $f(x)$ satisfies Eq. (62). The resulting equation for $g(z)$ has an analytic solution in terms of Kummer functions (Abramowitz and Stegun, 1974).

$$
\begin{gathered}
g(z)=C_{1} z \operatorname{Kummer} M\left(v_{1}, \frac{3}{2}, 2 \alpha^{2} z^{2}\right) \\
+C_{2} z \operatorname{Kummer} U\left(v_{1}, \frac{3}{2}, 2 \alpha^{2} z^{2}\right)
\end{gathered}
$$

where $v_{1}=\frac{4 \alpha^{2}+\omega^{2}}{8 \alpha^{2}}$.

For $\mu=0$ the equation for the perturbation $\eta$ is

$\eta_{z z}-4 \alpha^{2} z \eta_{z}-\alpha^{2}\left(\eta_{z}\right)^{2}+\eta\left(\frac{N^{2}}{z}+4 \alpha^{2}\right)=0$.

Applying the transformation Eq. (80) to Eq. (85) with $b=$ $e^{-1}$ and omitting the nonlinear term in $\phi^{2}$, we obtain for $\phi$ 
the same equation as Eq. (82) without the derivatives with respect to $x$. A particular solution of this equation is given by Eq. (83), while the solution of the homogeneous equation is

$\phi(z)=c_{1} \operatorname{erf}(i \sqrt{2} \alpha z)+c_{2}$

where $c_{1}$ and $c_{2}$ are constants.

\section{Summary and conclusions}

Computing numerical solutions for Long's equation has always been a challenge, even in some (singular) limiting cases. In this paper we introduced a transformation of this equation which under mathematically acceptable approximations leads to analytic expressions for the solutions. In particular, these solutions capture the dependence of the wave amplitude on the height.

The paper also provides an extension of Long's equation to the case where the atmospheric flow is not isothermal. This new equation can be solved analytically by the same transformation that is used for Long's equation.

Data availability. The MATLAB programs used to generate the plots in this paper are available from the author upon request.

Competing interests. The author declares that he has no conflict of interest.

Acknowledgements. The author would like to thank the editor and referees for their comments during the review process.

Edited by: Harindra Joseph Fernando

Reviewed by: two anonymous referees

\section{References}

Abramowitz, M. and Stegun, I.: Handbook of Mathematical Functions, Dover Publications, New York, 1974.

Akylas, T. R. and Davis, K. S.: Three-dimensional apects of nonlinear stratified flow over topography near the hydrostatic limit, J. Fluid Mech., 428, 81-105, 2001.

Baines, P. G.: Topographic effects in Stratified flows, Cambridge Univ. Press, New York, 1995.

Drazin, P. G.: On the steady flow of a fluid of variable density past an obstacle, Tellus, 13, 239-251, 1961.

Drazin, P. G. and Moore, D. W.: Steady two dimensional flow of fluid of variable density over an obstacle, J. Fluid. Mech., 28, 353-370, 1967.

Dubreil-Jacotin, M. L.: Complement a une note anterrieure sur les ondes de type permanent dans les liquides hterog'nes, Atti Accad. Lincei, Rend. Cl. Sci. Fis. Mat. Nat., 21, 344-366, 1935.
Durran, D. R.: Two-Layer solutions to Long's equation for vertically propagating mountain waves, Q. J. Roy. Meteor. Soc., 118, 415-433, 1992.

Fritts, D. C. and Alexander, M. J.: Gravity wave dynamics and effects in the middle atmosphere, Rev. Geophys., 41, 1003, https://doi.org/10.1029/2001RG000106, 2003.

Geller, M. A., Alexander, M. J., Love, P. T., Bacmeister, J., Ern, M., Hertzog, A., Manzini, E., Preusse, P., Sato, K., Scaife, A. A., and Zhou, T.: A comparison between gravity wave momentum fluxes in observations and climate models, J. Climate, 26, 6383-6405, 2013.

Humi, M.: On the Solution of Long's Equation Over Terrain, Il Nuovo Cimento C, 27, 219-229, 2004.

Humi, M.: On the Solutions of Long's Equation with Shear SIAM, J. Appl. Math., 66, 1839-1852, 2006.

Humi, M.: Density representation of Long's equation, Nonlin. Processes Geophys., 14, 273-283, https://doi.org/10.5194/npg-14273-2007, 2007.

Humi, M.: Long's equation in terrain following coordinates, Nonlin. Processes Geophys., 16, 533-541, https://doi.org/10.5194/npg16-533-2009, 2009.

Humi, M.: The effect of shear on the generation of gravity waves, Nonlin. Processes Geophys., 17, 201-210, https://doi.org/10.5194/npg-17-201-2010, 2010.

Humi, M.: Time-dependent Long's equation, Nonlin. Processes Geophys., 22, 133-138, https://doi.org/10.5194/npg-22-1332015, 2015.

Jumper, G. Y., Murphy, E. A., Ratkowski, A. J., and Vernin, J.: Multisensor Campaign to correlate atmospheric optical turbulence to gravity waves, AIAA paper AIAA-2004-1077, 42nd AIAA Aerospace Sciences Meeting and Exhibit Reno, Nevada, USA, 2004.

Lily, D. K. and Klemp, J. B.: The effect of terrain shape on nonlinear hydrostatic mountain waves, J. Fluid Mech., 95, 241-261, 1979.

Long, R. R.: Some aspects of the flow of stratified fluids I. Theoretical investigation, Tellus, 5, 42-57, 1953.

Long, R. R.: Some aspects of the flow of stratified fluids II. Theoretical investigation, Tellus, 6, 97-115, 1954.

Long, R. R.: Some aspects of the flow of stratified fluids III.Continuous density gradients, Tellus, 7, 341-357, 1955.

Long, R. R.: The motion of fluids with density stratification, J. Geophys. Res., 64, 2151-2163, 1959.

Miglietta, M. M. and Rotunno, R.: Numerical Simulations of Sheared Conditionally Unstable Flows over a Mountain Ridge, J. Atmos. Sci., 71, 1747-1762, 2014.

Nappo, C. J.: Atmospheric Gravity Waves, 2nd Edn., Academic Press, Boston, 2012.

Peltier, W. R. and Clark, T. L.: Nonlinear mountain waves in two and three spatial dimensions, Q. J. Roy. Meteor. Soc., 109, $527-$ 548, 1983.

Richter, J. H., Sassi, F., and Garcia, R. R.: Toward a physically based gravity wave source parameterization in a general circulation model, J. Atmos. Sci., 67, 136-156, 2010.

Shutts, G. J., Kitchen, M., and Hoare, P. H.: A large amplitude gravity wave in the lower stratosphere detected by radiosonde, Q. J. Roy. Meteor. Soc., 114, 579-594, 1988.

Shutts, G. J., Healey, P., and Mobbs, S. D.: A multiple sounding technique for the study of gravity waves, Q. J. Roy. Meteor. Soc., 120, 59-77, 1994. 
Smith, R. B.: Linear theory of stratified hydrostatic flow past an isolated mountain, Tellus, 32, 348-364, 1980.

Smith, R. B.: Hydrostatic airflow over mountains, Adv. Geophys., 31, 1-41, 1989.

Smith, R. B. and Kruse, G. K.: Broad-Spectrum Mountain Waves, J. Atmos. Sci., 74, 1381-1402, 2017

Vernin, J., Trinquet, H., Jumper, G., Murphy, E., and Ratkowski, A.: OHP02 gravity wave campaign in relation to optical turbulence, J. Environ. Fluid Mech., 7, 371-382, 2007.
Yih, C.-S.: Equations governing steady two-dimensional large amplitude motion of a stratified fluid, J. Fluid Mech., 29, 539-544, 1967.

Yih, C.-S.: Stratified flows, Academic Press, New York, NY, 1980. 